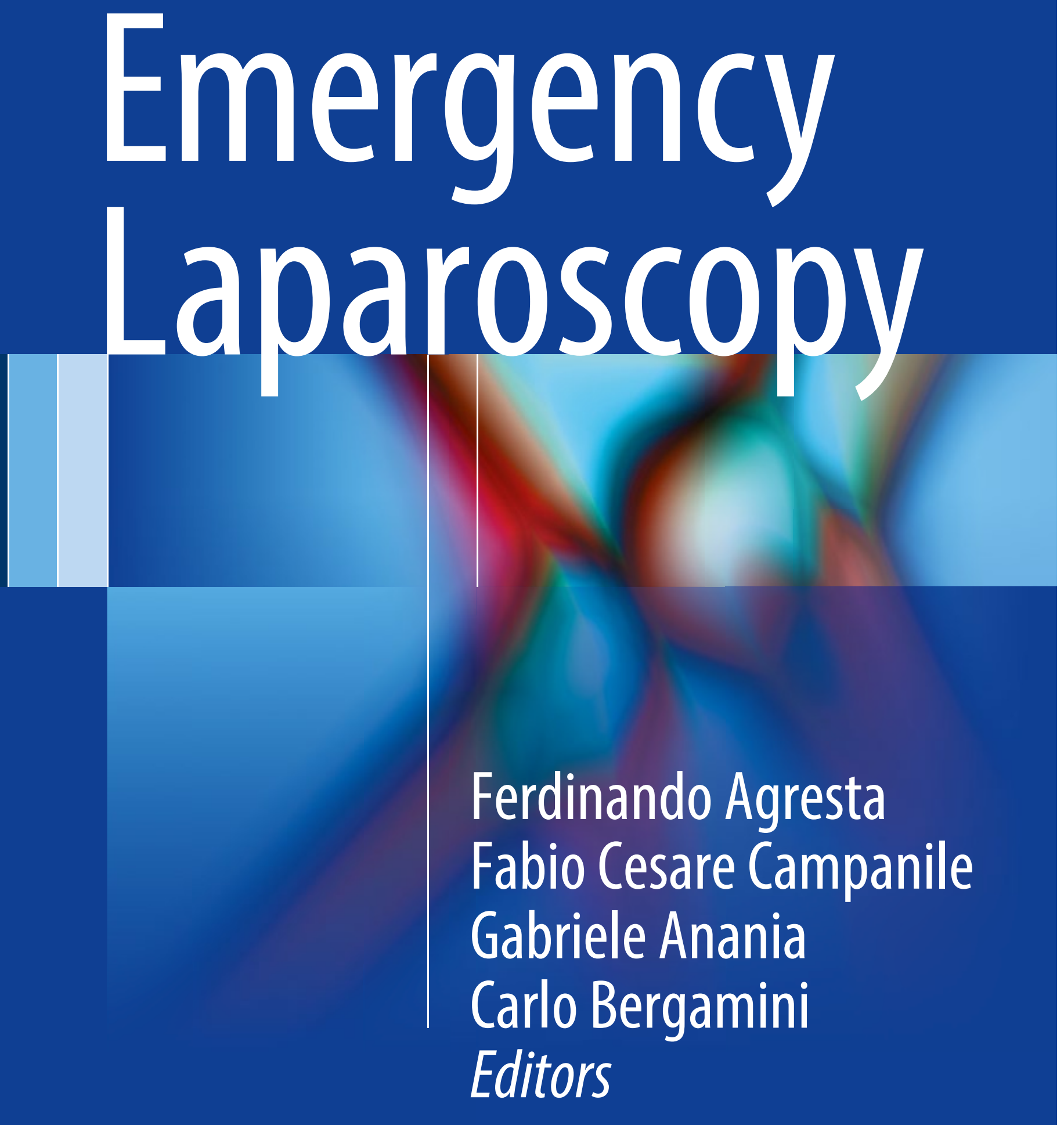




\section{Contents}

1 A Worldwide Overview of Emergency Laparoscopic Procedures..... 1

Carlo Bergamini, Ferdinando Agresta,

Fabio Cesare Campanile, and Gabriele Anania

2 Acute Calculous Cholecystitis ................................ 7

Fabio Cesare Campanile, Domenico Giannotti,

Ferdinando Agresta, Nereo Vettoretto, and Rao Ivatury

3 Acute Pancreatitis

Mario Campli, Alessandra Cerioli, Ari Leppäniemi,

Alberto Arezzo, and Carlo Bergamini

4 Laparoscopic Appendectomy

Nereo Vettoretto, Sarah Molfino, Giulia Montori,

Fabio Cesare Campanile, and Mauro Zago

5 Laparoscopy in Gynecologic Emergencies

Federico Coccolini, Giuseppe D’Amico, Giulia Montori,

Fausto Catena, Gustavo Fraga, and Luca Ansaloni

6 Nonspecific Abdominal Pain

Michele Carlucci, Aldo Alberto Beneduce, Guido Fiorentini, and Giovanni Burtulo

7 Perforated Gastroduodenal Ulcer

Antonino Mirabella, Massimo Lupo, Ferdinando Agresta,

Stefano Mandalà, Gabriele Anania, Mario Campli, and Kjetil Soreide

8 Emergency Lapararoscopy for Colon Obstruction and Acute Diverticulitis .

Alberto Arezzo, Marco E. Allaix, Mario Campli,

Carlo Bergamini, and Ferdinando Agresta

9 Laparoscopy in Small Bowel Obstruction

Mauro Zago, Diego Mariani, Hayato Kurihara,

Gianluca Baiocchi, Nereo Vettoretto, Carlo Bergamini,

Fabio Cesare Campanile, and Ferdinando Agresta 
10 Incarcerated Hernias ..................................... 137

Giovanni Alemanno, Riccardo Somigli, Paolo Prosperi,

Mario Campli, Alberto Arezzo, Andrea Valeri,

Carlo Bergamini, and Mauro Zago

11 Incarcerated Incisional and Ventral Hernias

Micaela Piccoli, Grazia Maria Attina', Vincenzo Trapani,

Domenico Marchi, Barbara Mullineris, and Bruno Scotto

12 Emergency Laparoscopy for Abdominal Trauma

Salomone Di Saverio, Arianna Birindelli, Andrea Biscardi,

Matteo Mandrioli, Luca Di Donato, Antonio Tarasconi,

Roberto Cirocchi, Carlo Coniglio, Giovanni Gordini, and Gregorio Tugnoli

13 Update in Laparoscopic Approach to Acute Mesenteric Ischemia.... 179

Gianfranco Cocorullo, Nicola Falco, Tommaso Fontana,

Roberta Tutino, Giuseppe Salamone, and Gaspare Gulotta

14 Anaesthesia and Emergency Laparoscopy...

Riccardo Ragazzi, Marco Tartaglione, Savino Spadaro,

Antonio Gioia, and Carlo Alberto Volta

15 Imaging in Emergency Surgery

Claudia Rossi, Mariateresa Martino, Roberto Ronza,

Roberto Rizzati, and Mariano Scaglione

16 Emergency Laparoscopy: The Nurse's Point of View

Monica Rolfo, Serena Langella, Valeria Esposito,

Antonio Valenti, Angela Di Marco, and Alessandro Ferrero

17 Emergency Laparoscopy in the Elderly

Gian Luca Baiocchi, Luca Arru, Federico Gheza,

Carlo Bergamini, Gabriele Anania, Fabio Cesare Campanile,

Mauro Zago, Nazario Portolani, and Ferdinando Agresta 


\title{
Update in Laparoscopic Approach to Acute Mesenteric Ischemia
}

\author{
Gianfranco Cocorullo, Nicola Falco, Tommaso Fontana, \\ Roberta Tutino, Giuseppe Salamone, and Gaspare Gulotta
}

\subsection{Introduction}

The laparoscopic approach to Acute Mesenteric Ischemia is today unclear and less debated (AMI).

There are in fact no clinical evidences on this particular focus and only few articles can be found in several databases (pubmed, cochrane library, etc.), and the problem concerns both diagnostic and therapeutic utilization of the procedure.

These considerations were already taken into account in 2012 EAES guidelines where the Grade of Recommendation (GoR) of laparoscopy in AMI was low in both diagnostic and therapeutic aspects.

According to the new Oxford Classification [1], the use of laparoscopy in patients with suspicious or diagnosed AMI presents a weak GoR.

\subsection{Epidemiology}

AMI is an uncommon but serious disease often associated with a bad prognosis, accounting $<1$ in every 1000 hospital admissions. Women are more affected than men with 3:1 ratio. Epidemiological study attributes this difference to higher longevity of females vs. males. Clinical risk factors may provide some clues for AMI diagnosis. Patients with a history of atrial fibrillation, recent myocardial infarction, congestive heart failure, or peripheral arterial embolism have an higher risk for Superior Mesenteric Artery (SMA) embolism. History of postprandial abdominal pain, weight loss, and food intolerance in patients with acute abdominal

G. Cocorullo $(\bowtie) \bullet$ N. Falco $\bullet$ T. Fontana $\bullet$ R. Tutino $\bullet$ G. Salamone $\bullet$ G. Gulotta Department of Surgical Oncological and Stomatological Sciences, Unit of General and Emergency Surgery, University of Palermo, Palermo, Italy

e-mail: gianfranco.cocorullo@unipa.it 
pain could indicate an acute thrombotic occlusion of SMA. AMI in fact is associated with occlusion of SMA for arterial embolism or thrombosis in $67.2 \%$ of cases, while mesenteric venous thrombosis occurs in $15.7 \%$. Only in remaining patients it is possible to find the third form of AMI without vessel obstruction, the so-called nonocclusive mesenteric ischemia (NOMI) [2].

\subsection{Diagnosis}

The diagnosis of AMI represent a very difficult diagnostic enigma; clinical markers are often aspecific but according to the literature severe and diffuse abdominal pain, bowel emptying, risk factor of embolism, arterial or venous thrombosis, and history of prior undiagnosed attacks of abdominal angina associated with specific laboratory tests (persistent $\mathrm{pH}<7.34$; plasmatic lactate level: $2.1 \mathrm{mmol} / \mathrm{L}, \mathrm{WBC}: 12,000 / \mathrm{mmc}$ ) could be highly suggestive of AMI [3].

According to the EAES guidelines and the latest articles of International literature, the gold standard for diagnosis of AMI is multidetector CT Angiography (CTA) with a sensibility of $93.3 \%$ and a specificity of $95.9 \%$ (Fig. 13.1) [4, 5].

In a recent review of more than 6000 patients, Acosta [5] underlines the secondary role of explorative laparotomy/laparoscopy to confirm cases of AMI without signs of SMA occlusion at CTA. Through the abdominal exploration, in fact, the surgeon could notice bowel aspect, its dilatation and peristaltism, the presence of evident pulsations in the mesenteric arcade arteries, and the bleeding from cut surfaces; the surgeon, moreover, could observe bowel wall necrosis and/or peritonitis in late form of AMI (Fig. 13.2).

Only few additional reports have been found on the diagnostic role of explorative laparoscopy. The laparoscopic diagnosis of AMI is clearly influenced by the stage of bowel damage, the etiology of the ischemia, and the experience of the surgeon. In fact, the ability to recognize signs of bowel ischemia depends on the

Fig. 13.1 CT angiography

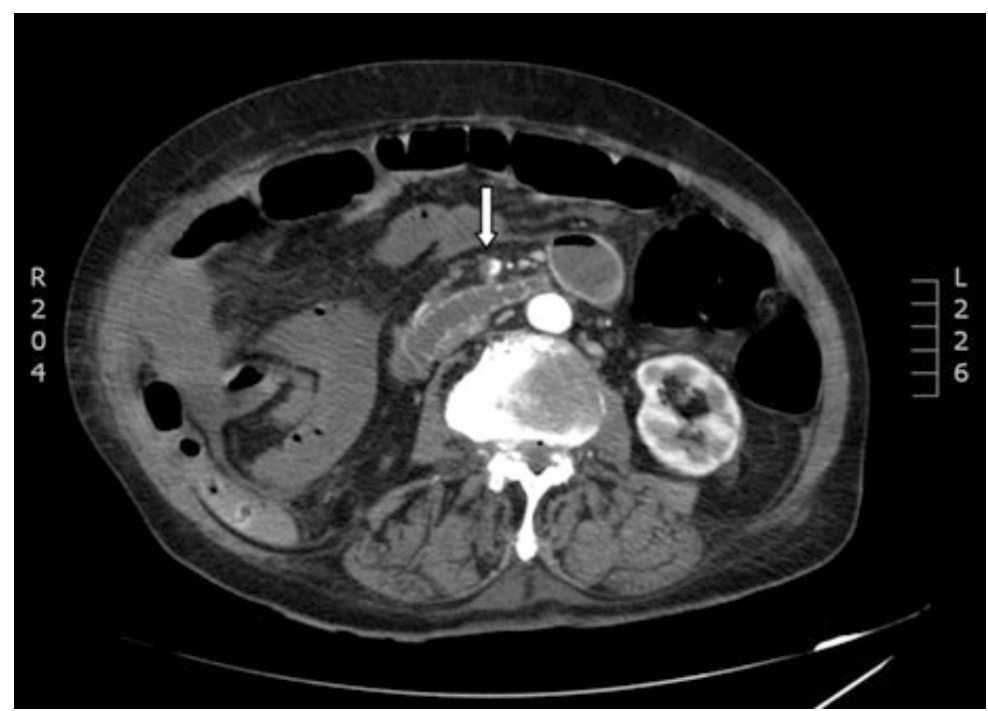


Fig. 13.2 Ischemic bowel

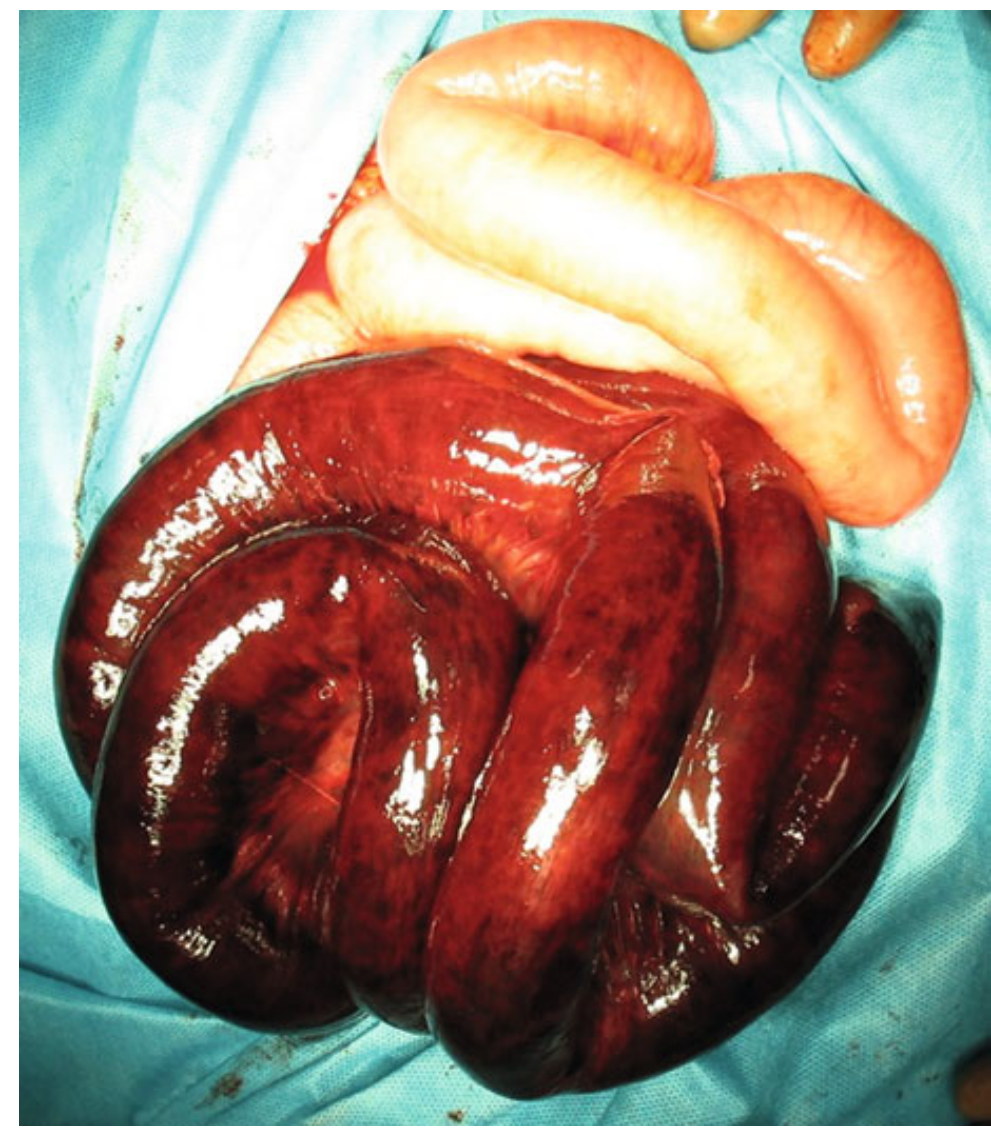

Fig. 13.3 Laparoscopic abdominal exploration

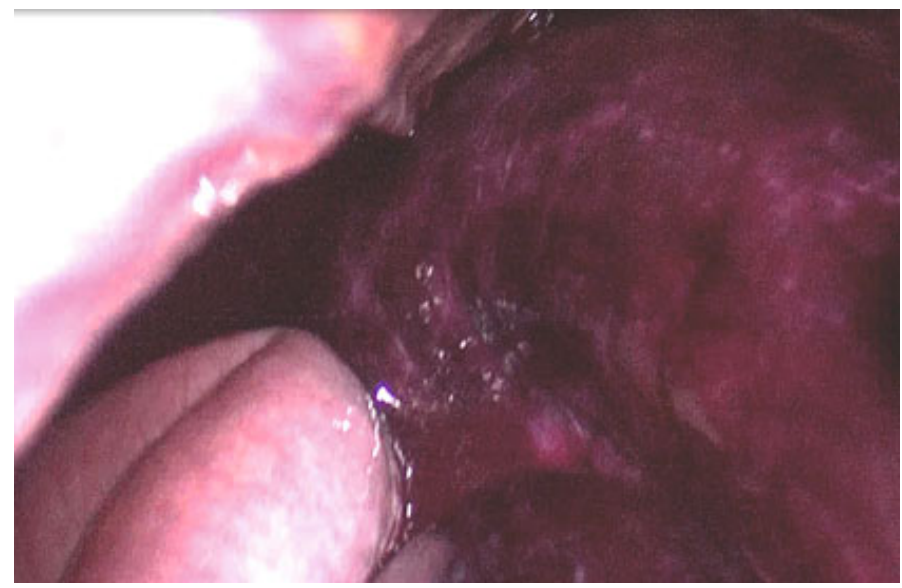

single operator's specific medical records and on the level of his learning curve, considering also that a low pressure of $\mathrm{CO}_{2}$ pneumoperitoneum is advised if there is a suspicious AMI (Fig. 13.3).

In this way, since the first application of fluorescein proposed in 1993 by Kam and Scheeres to increase the sensibility of laparoscopy, several studies on canine or porcine models were proposed [6]. However, only few evidences are collected from these studies and in particular Paral Jiri et al. (2007) confirmed on porcine model the feasibility of this procedure using the specific optical system in the follow-up of AMI patients. Moreover, there are no trials or perspective studies on humans. The article of Paral represents the last important experience in the use of fluorescein, but 
the related level of evidence (LE) in the EAES guidelines, which was $2 \mathrm{~b}$ in 2012, has been changed to LE 3 according to 2011 Oxford classification [7]. Unfortunately, in the last 2 years no studies have been produced on the primary diagnostic role of the laparoscopy and so the procedure maintains a weak GoR.

Most studies, instead, underline the possible role of laparoscopy with or without fluorescein in second-look exploration as "damage control surgery" [3, 5]. Nevertheless, in 2012, was published a study [3] that underlines the feasibility of a diagnostic laparoscopy in AMI in patients with Aortic Dissection (AoD). According to Tshomba $\mathrm{Y}$ et al that in patients with suspicious clinical signs of AMI without peritonitis if there are no conditions for rapid execution of CTA or a previous CTA had been not conclusive, an early diagnosis might be better than a clearer but late diagnosis. In this way, laparoscopy can reduce the number of unnecessary laparotomies overall in elderly critically ill patients. The study of Tshomba et al. [3] confirms that the best of laparoscopy in AMI diagnosis is the second look with the possibility of bedside use (directly in ICU). The author observed 202 consecutive AoD treated in the same center (71 acute type A AoD; 131 acute and chronic type B AoD) and only in 17 patients AMI was suspected. Diagnostic laparoscopy (DL) was performed in nine cases, in three cases during the medical treatment and in six patients after surgical or endovascular revascularization. In Tshomba's experience eight of nine DL were negative, but in one case AMI was diagnostic and the patient underwent revascularization in emergency. This experience is important, but it is based on few patients and so more studies need to confirm the feasibility and safeness of DL in AMI secondary to AoD; according to Tshomba the diagnostic power of DL increases in the second look overall in patients with aortic dissection type B (chronic type). In these cases, in fact, usually little time has been spent between starting of symptoms related to ischemic event and the evidence of bowel wall ischemia; therefore, it is known that sensitivity of diagnostic laparoscopy increases 24 hours after ischemic event. Moreover, according to the same article laparoscopy may be a feasible alternative to CTA in patients with kidney failure or other contraindications to injection of iodate CT contrast medium [3].

\subsection{Treatment}

Wyers in 2010 underlines that the first treatment of AMI is bowel revascularization. The second step is the reassessment of bowel viability. If possible the time of 20 or 30 min after revascularization should be spent before decision making about viability to evaluate signs of adequate perfusion as mesenteric vessel pulsation, normal color and appearance of the bowel serosa, peristalsis, and bleeding from cut surfaces [2]. Moreover, if an explorative diagnostic laparotomy (or laparoscopy) is performed as the first diagnostic step, an on-table SMA angiography should be performed at the same time. If no vascular surgeon is available, resection of obvious necrotic bowel should be performed and after the abdomen closure the patient should be transported to a vascular surgical center [5]. Main techniques to resolve the SMA occlusion are open SMA embolectomy for SMA embolism; other therapeutic options are 
hybrid or endovascular stenting in acute thrombotic occlusions, medical therapy with vasodilators and high dose of EBPM for thrombotic case, NOMI, and venous infarction [2].

In a limited number of cases when primary laparoscopy access is feasible and revascularization has been performed with endovascular access, it is possible to evaluate bowel perfusion laparoscopically and to perform at the same time a laparoscopical bowel resection of residual ischemic segments. The possibility of performing a "one-step anastomosis" will be evaluated each time by the surgeon, depending on the bowel presentation and the performance status (ASA score) of the patient. In nonpermissive patients, the surgeon should choose a two-step operation differing in performing anastomosis [8]. However, as suggested by the experience of Meriggi $\mathrm{F}$ et al. in their recent (2011) case report, laparoscopic primary access overall in AoD is an important tool for leading therapeutic decision and timing [9] (LE 4). It is clear that unfortunately there are no strong evidences about the therapeutic role of laparoscopy in AMI because only few experiences are published referring to a single center or more frequently to a single case report. More resources and efforts are needed in the evaluation of laparoscopic usefulness in AMI.

\subsection{Follow-Up and Damage Control Surgery}

Following the rule of damage control surgery a CTA is indicated for patients who underwent a stent positioning and it should be performed after the revascularization. The monitoring of restored bowel viability (second look) should be repeated two times in the postoperative time. Moreover, the second look is frequently performed also after surgical resection of necrotic bowel because an extension of ischemia is not rare in these patients and it requires further bowel resection. According to the literature in both cases a laparoscopic close exploration should represent a valid and more feasible technique than to perform an unsutured abdomen closure (Bogotà Bag, VA system, etc.) [5].

\subsection{Conclusions}

The gold standard for the primary diagnosis is CTA and laparoscopy has a limited rule. Fluorescein does not increase significantly the sensibility and specificity of the laparoscopy. The necessity to perform a low-pressure laparoscopy limits the diagnostic power of the procedure and reduces the feasibility and the diffusion of its execution. Nevertheless, the literature underlines the feasibility of a diagnostic laparoscopy in AMI after AoD when in the presence of suggestive clinical signs of AMI without peritonitis there are no conditions for the rapid execution of CTA or when a previous CTA had been nonconclusive. Laparoscopy may result in a feasible alternative to CTA also in patients with IRC that contraindicates injection of iodate CT contrast medium. Furthermore, laparoscopic primary access overall in AoD is an important tool for leading therapeutic decision and timing. The main rule of 
laparoscopy in AMI is the second look, in fact it should represent an alternative to multiple laparotomic explorations with less operation time and maintaining the closure of the abdomen. Another possible application of laparoscopy in the management of patient with AMI is the so-called bedside laparoscopy that for patients in ICU consents the repeatability of the procedure to follow the clinical course of patients. Referring to the therapeutic aspect, the usefulness of laparoscopy in AMI appears today limited only to few cases.

\section{References}

1. OCEBM Levels of Evidence Working Group. The Oxford levels of evidence 2. Oxford Centre for Evidence-Based Medicine, Oxford

2. Wyers MC (2010) Acute mesenteric ischemia: diagnostic approach and surgical treatment. Semin Vasc Surg 23(1):9-20

3. Tshomba Y, Coppi G, Marone EM, Bertoglio L, Kahlberg A, Carlucci M, Chiesa R (2012) Diagnostic laparoscopy for early detection of acute mesenteric ischaemia in patients with aortic dissection. Eur J Vasc Endovasc Surg 43(6):690-697

4. Agresta F, Ansaloni L, Baiocchi GL, Bergamini C, Campanile FC, Carlucci M, Cocorullo G, Corradi A, Franzato B, Lupo M, Mandalà V, Mirabella A, Pernazza G, Piccoli M, Staudacher C, Vettoretto N, Zago M, Lettieri E, Levati A, Pietrini D, Scaglione M, De Masi S, De Placido G, Francucci M, Rasi M, Fingerhut A, Uranüs S, Garattini S (2012) Laparoscopic approach to acute abdomen from the Consensus Development Conference of the Società Italiana di Chirurgia Endoscopica e nuove tecnologie (SICE), Associazione Chirurghi Ospedalieri Italiani (ACOI), Società Italiana di Chirurgia (SIC), Società Italiana di Chirurgia d'Urgenza e del Trauma (SICUT), Società Italiana di Chirurgia nell'Ospedalità Privata (SICOP), and the European Association for Endoscopic Surgery (EAES). Surg Endosc 26(8):2134-2164

5. Acosta S (2014) Surgical management of peritonitis secondary to acute superior mesenteric artery occlusion. World J Gastroenterol 20(29):9936-9941

6. Kam DM, Scheeres DE (1993) Fluorescein-assisted laparoscopy in the identification of arterial mesenteric ischemia. Surg Endosc 7(2):75-78

7. Paral J, Ferko A, Plodr M, Raupach J, Hadzi-Nikolov D, Dolezal D, Chovanec V (2007) Laparoscopic diagnostics of acute bowel ischemia using ultraviolet light and fluorescein dye: an experimental study. Surg Laparosc Endosc Percutan Tech 17(4):291-295

8. Palanivelu CI, Rangarajan M, Maheshkumaar GS, Rajan PS (2008) Relaparoscopy in the management of acute abdomen due to localized ischemic bowel: a novel technique-case report. Int J Surg 6(6):e89-e91

9. Meriggi F, Alloni A, Gramigna P, Tramelli P, Vigano M (2011) Acute aortic dissection with intestinal ischemia: what to do first. Ann Thorac Cardiovasc Surg 17(6):631-633 